\title{
Preditores de recorrência e progressão do câncer de bexiga: validação externa do escore de risco EORTC e a Classificação do Instituto do Câncer do Estado de São Paulo
}

\author{
Predicting bladder cancer recurrence and progression: EORTC risk score \\ external validation and the Sao Paulo Cancer Institute Classification
}

\author{
André Torreão Machadoํㅜ, Ricardo Waksman', Daher Cezar Chade², \\ Maurício Dener Cordeiro², Álvaro Sadek Sarkis², William Carlos Nahas ${ }^{3}$
}

\begin{abstract}
Machado AT, Waksman R, Chade DC, Cordeiro MD, Sarkis AS, Nahas WC. Preditores de recorrência e progressão do câncer de bexiga: validação externa do escore de risco EORTC e a Classificação do Instituto do Câncer do Estado de São Paulo / Predicting bladder cancer recurrence and progression: EORTC risk score external validation and the Sao Paulo Cancer Institute Classification. Rev Med (São Paulo). 2017 abr.-jun.;96(2):88-93.

RESUMO: Introdução: Classificações de risco baseadas em fatores preditivos de recorrência e progressão são essenciais para condutas no câncer de bexiga. Tabelas de risco combinam essas variáveis para uso clínico. As tabelas de risco da Organização Europeia para Pesquisa e Tratamento do Câncer (EORTC) são aceitas para esse propósito, mas nunca foram validadas no Brasil. Objetivos: Validar as tabelas de risco EORTC e criar uma classificação de risco baseada na população de pacientes acompanhados em um centro terciário de câncer. Métodos: Estudo retrospectivo de 561 pacientes submetidos a ressecção transuretral (RTU) de câncer de bexiga superficial de fevereiro de

escore $3=20,7 \%$; escore $4=29,2 \%$; escore $7=37,9 \%$. Para prever recorrência e progressão, estadio T e tamanho do tumor previram significativamente o desfecho. A classificação em escores foi: $\mathrm{Ta} /$ $\mathrm{CIS}=0$ pontos, $\mathrm{T} 1=2$ pontos, tamanho do tumor $=0$ ou 2 pontos . A classificação de risco foi obtida somando os pontos. A taxa de recorrência em 2 anos foi: escore $0=17 \%$; escore $2=28,6 \%$; escore $4=40,7 \%$. Conclusões: Constatamos que as tabelas de risco EORTC não conseguiram prever recorrência ou progressão do câncer de bexiga na nossa população. Portanto, desenvolvemos uma classificação de risco para auxiliar urologistas a individualizar as condutas por paciente.
\end{abstract} 2005 a junho de 2011. As variáveis analisadas foram as mesmas das tabelas de risco EORTC. A regressão logística foi realizada usando SPSS. A análise da curva COR determinou o limite de tamanho do tumor. Resultados: As tabelas de risco EORTC não conseguiram prever recorrência nem progressão. Na análise para prever recorrência isoladamente, estadio $\mathrm{T}$ e tamanho do tumor previram o desfecho. O limite de tamanho do tumor foi definido em $<4 \mathrm{~cm}$ vs $\geq 4 \mathrm{~cm}$ ( $\mathrm{AUC}=0,61 ; \mathrm{p}=0,001$ ). Criamos uma classificação: $\mathrm{Ta} / \mathrm{CIS}=0$ pontos, $\mathrm{T} 1=4$ pontos, tamanho do tumor $=0$ ou 3 pontos. A classificação de risco foi obtida somando os pontos. A taxa de recorrência em 2 anos foi: escore $0=11,2 \%$;
Descritores: Institutos de câncer; Neoplasias da bexiga urinária; Recidiva local de neoplasia/classificação; Estudos de validação; Brasil.

ABSTRACT: Introduction: Risk classification based on predictive factors of bladder cancer recurrence and progression is essential for management decision. Risk tables combine these variables for clinical practice use. European Organization for Research and Treatment of Cancer (EORTC) risk tables are widely accepted for this purpose, however they were never

Premio ABLAC 2017. Apresentado no III Congresso da Associação Brasileira das Ligas Acadêmicas de Cirurgia, São Paulo, SP, 2017. 1. Estudante da Faculdade de Medicina da Universidade de São Paulo. Email: andretm23@gmail.com; ricardo.waksman@gmail.com.

2. Médico Assistente da Clínica Urológica do Hospital das Clínicas da Faculdade de Medicina da Universidade de São Paulo. email: dchade@gmail.com,mauriciocordeiro99@yahoo.com, alsarkis@uol.com.br;

3. Professor Titular de Urologia da Faculdade de Medicina da USP. Instituto do Câncer do Estado de São Paulo (ICESP). Email: wnahas@uol.com.br.

Endereço para correspondência: André Torreão Machado. Rua Afonso Celso, 694 Apto. 81 B1. B. São Paulo, SP, Brasil. CEP: 04119-060. 
validated in Brazil. Our aim was to validate the EORTC risk tables and create a risk classification based on our population. Methods: Retrospective study of 561 patients who underwent transurethral resection of superficial bladder from February 2005 to June 2011. Variables analyzed were the same as EORTC risk tables. Logistic regression was performed using SPSS. ROC curve analysis was used for determining the cut-off for tumor size. Results: EORTC risk tables were not able to predict neither disease recurrence nor progression. In our analysis for predicting bladder cancer recurrence alone, we found that $\mathrm{T}$ stage and tumor size predicted outcome. Tumor size cut-off was defined as $<4 \mathrm{~cm}$ $\mathrm{vs} \geq 4(\mathrm{AUC}=0.61 ; \mathrm{p}=0.001)$. We created a scoring classification: $\mathrm{Ta} / \mathrm{CIS}=0$ points, $\mathrm{T} 1=4$ points, tumor size $=0$ or 3 points. Risk classification was obtained by adding the points accordingly and the following recurrence rate at 2 yrs by group: score $0=11.2 \%$;

\section{INTRODUÇÃO}

câncer de bexiga é o nono tipo de câncer em incidência no mundo, com 430.000 novos casos diagnosticados em $2012^{1}$.

Muitos pacientes não morrem da doença, porém tem múltiplas recidivas ${ }^{2}$. A neoplasia de bexiga é mais comumente diagnosticada em indivíduos mais velhos, com idade mediana de diagnóstico de 69 anos em homens e 71 anos em pessoas do sexo feminino. Em homens idosos, o câncer de bexiga é a segunda doença maligna mais prevalente, após o câncer de próstata ${ }^{2,3,4}$.

O tabagismo é o fator de risco mais importante para o câncer de bexiga. $O$ risco em fumantes é de 2 a 6 vezes maior que em não-fumantes ${ }^{5}$.

A biologia e a história natural relacionadas ao câncer de bexiga não músculo invasivo são bem conhecidas ${ }^{6}$. O tipo histológico mais comum é o carcinoma urotelial.

Esquemas de estratificação de risco e um sistema de gradação simplificam a estimativa de probabilidades de recorrência e de progressão e guiam a decisão de condutas no tratamento da doença, o aconselhamento do paciente e a inclusão em estudos clínicos ${ }^{6,7}$.

Entretanto, a maioria das decisões clínicas em casos de câncer de bexiga são feitas sem levar em conta nenhuma ferramenta de predição ${ }^{8}$. A predição do desfecho da doença baseada somente na experiência do médico está sujeita a erros, devido à subjetividade desse método ${ }^{9,10}$.

O estadiamento TNM do Comitê Conjunto Americano sobre Câncer é útil para prever risco de recorrência da doença em pacientes submetidos a cistectomia radical $^{11}$. A classificação TNM permite a subdivisão do câncer de bexiga em câncer de bexiga não invasor da camada muscular (câncer de bexiga superficial, Ta ou T1), e câncer de bexiga invasor da camada muscular (T2, T3 ou T4). Porém, foi mostrado que esse sistema de estadiamento é menos preciso que modelos que utilizam diversos parâmetros clínicos pré e pós-operatórios de cada paciente, permitindo uma medicina individualizada ${ }^{7}$.

Com o objetivo de prever as probabilidades de recorrência do câncer de bexiga superficial, a Organização score $3=20.7 \%$; score $4=29.2 \%$; score $7=37.9 \%$. The statistical model for bladder cancer recurrence or progression found that $\mathrm{T}$ stage and tumor size predicted the outcome. The scoring classification was: $\mathrm{Ta} / \mathrm{CIS}=0$ points, $\mathrm{T} 1=2$ points, tumor size $=0$ or 2 points. Risk classification was obtained by adding the points accordingly and the following recurrence rate at 2 yrs by group: score $0=17 \%$; score $2=28.6 \%$; score $4=40.7 \%$. Conclusions: We found that EORTC risk tables could not predict bladder cancer recurrence or progression in our patient population, possibly due to differences in patient characteristics. Therefore, we developed a new risk classification to aid urologists to individualize the management decision per patient.

Keywords: Cancer care facilities; Urinary bladder neoplasms; Validation studies; Brazil.

Europeia para Pesquisa e Tratamento do Câncer (EORTC) desenvolveu um sistema de pontuação e tabelas de risco. ${ }^{12}$ Essa ferramenta foi elaborada a partir de dados de 2596 pacientes com câncer de bexiga superficial, e levou em conta os 6 mais importantes preditores clínicos e patológicos de desfecho: estadio T, grau histológico, número de tumores, tamanho do tumor, carcinoma in situ concomitante e história prévia de recorrência da doença ${ }^{7}$.

As tabelas de risco da EORTC são aceitas mundialmente e possuem validação externa ${ }^{13-18}$. No entanto, nunca foram validadas no Brasil.

\section{OBJETIVOS}

Validar as tabelas de risco EORTC e criar uma classificação de risco baseada na população de pacientes acompanhados em um centro de referência terciário de câncer.

\section{MATERIAIS E MÉTODOS}

Este trabalho trata-se de um estudo retrospectivo de 561 pacientes submetidos a ressecção trans-ureteral de câncer de bexiga não-invasor da camada muscular de fevereiro de 2005 a junho de 2011. As seguintes variáveis foram analisadas: sexo, idade, grau histológico do tumor, número de recorrências prévias, estadio pT (Ta, T1 ou Cis), quantidade das lesões e tamanho da lesão em centímetros. A regressão logística foi realizada usando SPSS.

A curva da Característica de Operação do Receptor (COR) foi utilizada para definir os limites para a variável tamanho da lesão.

Na variável estadio pT, nas análises para recidiva, foram agrupados os pacientes com Ta e Cis, após a análise estatística indicar que não havia diferença significativa entre as probabilidades de recidiva entre esses grupos.

Os desfechos estudados foram a recidiva em 2 anos de seguimento e a progressão da doença em 2 anos de seguimento.

A distribuição das características dos pacientes está mostrada na Tabela 1 . 
Tabela 1. Características dos pacientes

\begin{tabular}{|c|c|c|}
\hline \multicolumn{2}{|l|}{ Variáveis } & \multirow{2}{*}{$\begin{array}{l}\mathbf{N}^{0} \\
419\end{array}$} \\
\hline \multirow{2}{*}{ Sexo } & Masculino & \\
\hline & Feminino & 139 \\
\hline \multirow{2}{*}{ Idade } & $\begin{array}{l}\text { Sim } \\
\text { média }=65,4 \\
\text { desvio padrão }=11,6 \\
\text { valor mínimo }=22,0 \\
\text { valor máximo }=94,0\end{array}$ & 397 \\
\hline & $\begin{array}{l}\text { Não } \\
\text { média }=67,4 \\
\text { desvio padrão }=11,2 \\
\text { valor mínimo }=40,0 \\
\text { valor máximo }=93,0\end{array}$ & 161 \\
\hline \multirow{2}{*}{ Grau histológico } & Alto & 273 \\
\hline & Baixo & 282 \\
\hline \multirow{2}{*}{$\begin{array}{l}\text { Número de recorrências } \\
\text { prévias }\end{array}$} & 0 & 458 \\
\hline & $\geq 1$ & 96 \\
\hline \multirow{3}{*}{ Estadio pT } & $\mathrm{Ta}$ & 305 \\
\hline & Cis & 33 \\
\hline & $\mathrm{T} 1$ & 218 \\
\hline \multirow{2}{*}{ Quantidade de lesões } & $\begin{array}{l}\text { Sim } \\
\text { média }=2,5 \\
\text { desvio padrão }=3,0 \\
\text { valor mínimo }=1,0 \\
\text { valor máximo }=2,0\end{array}$ & 346 \\
\hline & $\begin{array}{l}\text { Não } \\
\text { média }=2,6 \\
\text { desvio padrão }=2,5 \\
\text { valor mínimo }=1,0 \\
\text { valor máximo }=12,0\end{array}$ & 125 \\
\hline \multirow{2}{*}{ Tamanho da lesão } & $\begin{array}{l}\text { Sim } \\
\text { média }=3,8 \\
\text { desvio padrão }=2,6 \\
\text { valor mínimo }=0,4 \\
\text { valor máximo }=15,0\end{array}$ & 339 \\
\hline & $\begin{array}{l}\text { Não } \\
\text { média }=4,8 \\
\text { desvio padrão }=2,9 \\
\text { valor mínimo }=0,0 \\
\text { valor máximo }=15,0\end{array}$ & 122 \\
\hline
\end{tabular}

\section{RESULTADOS}

As tabelas de risco EORTC não conseguiram prever nem recorrência nem progressão de doença na nossa população. $\mathrm{Na}$ análise univariada vários fatores da tabela de risco da EORTC não funcionaram como preditores na nossa população.

Seleção de variáveis para o modelo multivariado da Classificação do Instituto do Câncer do Estado de
São Paulo: Todas as variáveis analisadas no estudo foram primeiro sujeitas a uma análise univariada (teste qui-quadrado para sexo, grau histológico, número de recorrências prévias, estadio $\mathrm{pT}$, teste t-Student para idade e quantidade de lesões), e somente foram incluídas nas análises com modelo multivariado aquelas em que foi mostrado que há diferença entre os grupos.

Foram feitas 3 análises separadas dos parâmetros, a fim de encontrar os preditores para 3 situações: recorrência separadamente, recorrência em conjunto com progressão, e somente progressão.

Fatores prevendo recidiva sem progressão: Conforme indicado na Tabela 2, as seguintes variáveis têm importância para prognóstico na análise univariada: número de recorrências, estadio pT e tamanho da lesão.

Tabela 2. Análise univariada para recidiva

\begin{tabular}{l|c|c}
\hline \multirow{2}{*}{ Varíavel } & \multicolumn{2}{|c}{ Recidiva } \\
\cline { 2 - 3 } & OR & p \\
\hline Sexo & 0,90 & 0,65 \\
\hline Idade & 1,01 & 0,13 \\
\hline Grau histológico & 1,36 & 0,12 \\
\hline Número de recorrências prévias & 1,65 & 0,04 \\
\hline Estadio pT & 2,37 & 0,00 \\
\hline Número de lesões & & 0,91 \\
\hline Tamanho das lesões & 2,27 & 0,00 \\
\hline
\end{tabular}

Usando a curva COR encontrou-se que o tamanho da lesão é um preditor significativo para recidiva (área sob curva $-\mathrm{AUC}=0.616 \mathrm{e} \mathrm{p}<0.00$ ). O melhor limite obtido foi o de $<3,75 \mathrm{~cm}$ versus $\geq 3,75 \mathrm{~cm}$, com sensibilidade de $61,9 \%$ e especificidade de $58,4 \%$.

Portanto, as variáveis incluídas no modelo multivariado foram as seguintes: número de recorrências prévias $(0, \geq 1)$, estadio $\mathrm{pT}$ (Ta ou Cis, T1) e tamanho das lesões $(<3,75 \mathrm{~cm}, \geq 3,75 \mathrm{~cm})$, como mostrado na Tabela 3 .

Tabela 3. Análise multivariada para recidiva

\begin{tabular}{c|l|l|c}
\hline \multirow{2}{*}{} & \multirow{2}{*}{ Variável } & \multicolumn{2}{|c}{ Recidiva } \\
\cline { 3 - 4 } & OR (IC 95\%) & p \\
\hline \multirow{4}{*}{$1^{\circ}$ Passo } & $\begin{array}{l}\text { Número de } \\
\text { recorrências }\end{array}$ & $1,53(0,80 ; 2,93)$ & 0,19 \\
\cline { 2 - 4 } & Estadio pT & $2,38(1,46 ; 3,90)$ & 0,00 \\
\cline { 2 - 4 } & $\begin{array}{l}\text { Tamanho da } \\
\text { lesão }\end{array}$ & $1,91(1,16 ; 3,17)$ & 0,01 \\
\hline \multirow{2}{*}{$2^{\circ}$ Passo } & Estadio pT & $2,37(1,45 ; 3,88)$ & 0,00 \\
\cline { 2 - 4 } & $\begin{array}{l}\text { Tamanho da } \\
\text { lesão }\end{array}$ & $1,82(1,11 ; 2,99)$ & 0,01 \\
\hline
\end{tabular}


Machado AT, et al. Preditores de recorrência e progressão do câncer de bexiga: validação externa do escore.

No modelo final, apenas estadio pT e tamanho da lesão permaneceram estatisticamente significativos.

Baseado nos resultados das razões de chances, foi criado uma pontuação, em que quem tem Cis ou Ta recebe 0 pontos e quem tem $\mathrm{T} 1$ recebe 4 pontos. De maneira análoga, quem tem tamanho de lesão $<3,75 \mathrm{~cm}$ recebe 0 pontos e quem tem tamanho de lesão $\geq 3,75 \mathrm{~cm}$ recebe 3 pontos.

Logo, os pacientes podem ser divididos em 4 grupos, de acordo com a somatória das pontuações: 0 , 3, 4 ou 7, como representado na Tabela 4 .

Tabela 4. Probabilidade de recidiva de acordo com a pontuação total

\begin{tabular}{c|l|c}
\hline \multirow{2}{*}{ Pontuação } & \multicolumn{2}{|c}{ Recidiva } \\
\cline { 2 - 3 } & OR (IC 95\%) & $\mathrm{p}$ \\
\hline 0 & 1.00 & 0,00 \\
\hline 3 & $2,15(1,07 ; 4,32)$ & 0,03 \\
\hline 4 & $2,87(1,38 ; 5,97)$ & 0,00 \\
\hline 7 & $4,58(2,48 ; 8,46)$ & 0,00 \\
\hline
\end{tabular}

Logo, os pacientes com pontuação 3 têm chance 2,15 vezes maior de recidiva em 2 anos, aqueles com pontuação igual a 4 tem 2,87 vezes maior probabilidade de recidiva em 2 anos, e aqueles com pontuação 7, 4,58 maior probabilidade de recidiva em 2 anos.

Fatores prevendo recidiva com ou sem progressão: Na análise univariada, apenas o estadio pT e o tamanho da lesão apresentaram importância para prever recidiva com ou sem progressão. A Tabela 5 representa esses resultados.

Tabela 5. Análise univariada para recidiva com ou sem progressão

\begin{tabular}{l|c|c}
\hline \multirow{2}{*}{ Variável } & \multicolumn{2}{|c}{$\begin{array}{l}\text { Recidiva com ou sem } \\
\text { progressão }\end{array}$} \\
\cline { 2 - 3 } & OR & p \\
\hline Sexo & 1,10 & 0,64 \\
\hline Idade & 1,01 & 0,06 \\
\hline Grau histológico & 1,24 & 0,24 \\
\hline Número de recorrências prévias & 1,49 & 0,09 \\
\hline Estadio pT & 1,83 & 0,00 \\
\hline Número de lesões & & 0,75 \\
\hline Tamanho da lesão & 1,14 & 0,00 \\
\hline
\end{tabular}

Utilizando, de maneira semelhante à da análise para recidiva, a curva COR, foi mostrado que o tamanho da lesão é um preditor satisfatório para recidiva com ou sem progressão ( $\mathrm{AUC}=0,61$ e $\mathrm{p}<0,00)$. O melhor limite obtido foi $<3,75 \mathrm{~cm}$ versus $\geq 3,75 \mathrm{~cm}$, com sensibilidade de $60,7 \%$ e especificidade de $58,4 \%$.

Logo, foram incluídos no modelo multivariado estadio pT (Ta ou Cis, T1) e tamanho da lesão $(<3,75 \mathrm{~cm}$, $\geq 3,75 \mathrm{~cm}$ ), como está evidente na Tabela 6 .

Tabela 6. Análise multivariada para recidiva com ou sem progressão

\begin{tabular}{l|l|c}
\hline \multirow{2}{*}{ Variável } & \multicolumn{2}{l}{ Recidiva com ou sem progressão } \\
\cline { 2 - 3 } & OR (IC 95\%) & p \\
\hline Estadio pT & $1,76(1,13 ; 2,74)$ & 0,01 \\
\hline Tamanho da lesão & $1,89(1,22 ; 2,95)$ & 0,00 \\
\hline
\end{tabular}

Utilizando os resultados das razões de chances, foi criado uma pontuação, em que quem tem Cis ou Ta recebe 0 pontos e quem tem $\mathrm{T} 1$ recebe 2 pontos. De forma semelhante, um tamanho de lesão $<3,75 \mathrm{~cm}$ e um tamanho de lesão $\geq 3,75$, correspondem, respectivamente, a 0 e 2 pontos.

Logo, os pacientes podem ser divididos em 4 grupos, de acordo com a somatória das pontuações: 0,2 , ou 4, conforme mostrado na Tabela 7.

Tabela 7. Probabilidade de Recidiva com ou sem progressão de acordo com a pontuação total

\begin{tabular}{c|l|c}
\hline \multirow{2}{*}{ Pontuação } & \multicolumn{2}{|l}{ Recidiva com ou sem progressão } \\
\cline { 2 - 3 } & OR (IC 95\%) & p \\
\hline 0 & 1,00 & 0,00 \\
\hline 2 & $2,04(1,21 ; 3,45)$ & 0,00 \\
\hline 4 & $3,37(1,95 ; 5,81)$ & 0,00 \\
\hline
\end{tabular}

Logo, os pacientes com pontuação 2 têm chance 2,04 vezes maior de recidiva em 2 anos, aqueles com pontuação igual a 4 tem 3,37 vezes maior probabilidade de recidiva em 2 anos.

Fatores prevendo apenas progressão: $\mathrm{Na}$ análise univariada, apenas o tamanho da lesão se mostrou um bom preditor de progressão. Essa análise está representada na Tabela 8.

Tabela 8. Análise univariada para progressão

\begin{tabular}{l|c|c}
\hline \multirow{2}{*}{ Variável } & \multicolumn{2}{|c}{ Progressão } \\
\cline { 2 - 3 } & OR & p \\
\hline Sexo & 1,07 & 0,87 \\
\hline Idade & 1,02 & 0,15 \\
\hline Grau histológico & 0,83 & 0,64 \\
\hline Número de recorrências prévias & 0,86 & 0,79 \\
\hline Estadio pT T1 x Ta Cis & 0,50 & 0,15 \\
& 1,53 & 0,51 \\
\hline Número de lesões & 0,33 & 0,13 \\
\hline Tamanho da lesão & 1,17 & 0,01 \\
\hline
\end{tabular}


Como o tamanho da lesão foi a única variável significativa para prever progressão, não podemos realizar análise multivariada.

Usando a curva COR, foi mostrado que o tamanho da lesão é um preditor significativo de progressão (AUC $=0.62$ e $\mathrm{p}=0.03)$. O limite foi estabelecido em $<4,75$ $\mathrm{cm}$ versus $\geq 4,75 \mathrm{~cm}$, com sensibilidade de $52,0 \%$ e especificidade de $70,8 \%$.

Os pacientes foram então divididos em dois grupos de acordo com os resultados obtidos nessa análise, como mostra a Tabela 9.

Tabela 9. Probabilidade de progressão de acordo com o tamanho da lesão

\begin{tabular}{l|l|c}
\hline \multirow{2}{*}{ Tamanho da lesão } & \multicolumn{2}{|c}{ Progressão } \\
\cline { 2 - 3 } & OR (IC 95\%) & p \\
\hline$<4,75$ & 1,00 & 0,00 \\
\hline$\geq 4,75$ & $2,62(1,15 ; 5,95)$ & 0,02 \\
\hline
\end{tabular}

Logo, os pacientes com tamanho de lesão maior ou igual a 4,75 centímetros têm probabilidade 2,62 vezes maior de recidiva em 2 anos, quando comparados àqueles com tamanho de lesão menor que 4,75 centímetros.

Na Tabela 10 constam os pesos das 2 pontuações geradas, para uso clínico prático.

Tabela 10. Pesos para cálculo de recidiva

\begin{tabular}{|c|c|c|c|}
\hline \multicolumn{2}{|l|}{ Preditor } & \multirow{3}{*}{$\begin{array}{c}\text { Recidiva } \\
0\end{array}$} & \multirow{2}{*}{$\begin{array}{l}\begin{array}{l}\text { Recidiva } \\
\text { com ou sem } \\
\text { progressão }\end{array} \\
0\end{array}$} \\
\hline \multirow{3}{*}{ Estadio pT } & \multirow{3}{*}{$\begin{array}{l}\text { Ta ou Cis } \\
\mathrm{T} 1\end{array}$} & & \\
\hline & & & \\
\hline & & 4 & 2 \\
\hline \multirow{2}{*}{$\begin{array}{l}\text { Tamanho da } \\
\text { lesão }\end{array}$} & $\begin{array}{l}<3,75 \mathrm{~cm} \\
\geq 3,75 \mathrm{~cm}\end{array}$ & 0 & 0 \\
\hline & & 3 & 2 \\
\hline \multicolumn{2}{|c|}{ Pontuação total } & $0-7$ & $0-4$ \\
\hline
\end{tabular}

\section{DISCUSSÃO}

Os preditores encontrados para o câncer de bexiga superficial foram o estadio $\mathrm{T}$ o tamanho da lesão, fatores que já foram apontados nos estudos de validação externa da tabela de risco da EORTC como importantes para a classificação de risco do câncer de bexiga ${ }^{13-18}$.
O estadio T também foi levado em conta na criação da classificação de risco do The Club Urológico Español de Tratamiento Oncológico (CUETO) ${ }^{19}$. A classificação CUETO foi baseada no modelo da EORTC e também foi validada externamente ${ }^{20,21}$.

Não houve validação externa das tabelas de risco da EORTC, embora a evidência dos dois preditores desse estudo aponta para semelhanças na plausibilidade biológica da nossa classificação.

Seguindo o modelo das tabelas de risco da EORTC $^{12}$, a ferramenta mostrada neste estudo separa os desfechos recidiva e progressão, o que permitiria, na prática clínica diária, a individualização da decisão de condutas e facilitaria a comunicação médico-paciente. Para casos de tumores cuja pontuação aponte para grande probabilidade de recidiva, esse deve ser o foco da conversa e da decisão do tratamento. Já nos casos que mostrarem um risco maior de progressão da doença, é possível dar maior importância a esse evento.

Foi possível com esse estudo, a criação de uma ferramenta ajustada para a população brasileira e que utiliza critérios patológicos frequentemente obtidos no manejo do câncer de bexiga superficial, portanto teria especial utilidade na prática clínica urológica no Brasil.

Diferenças da população brasileira em relação às amostragens dos outros trabalhos poderiam explicar essa divergência nos resultados obtidos. Mais estudos são necessários para ampliar o conhecimento da comunidade científica nessa área.

Consideramos o tamanho da amostra a maior limitação do trabalho, o que pode explicar não ter sido possível validar as tabelas de risco EORTC para a população brasileira. Porém a validação pode ser conseguida se mais trabalhos similares a este forem desenvolvidos, incluindo uma população maior no estudo.

\section{CONCLUSÃO}

As tabelas de risco da EORTC não conseguiram prever recorrência nem progressão de câncer de bexiga superficial na nossa população, possivelmente devido a diferenças nos nossos pacientes comparados aos estrangeiros, ou a diferenças no sistema de saúde. O problema do sobreajuste também explica em parte a dificuldade da validação das tabelas de risco da EORTC no Brasil.

Contudo, criamos uma nova ferramenta preditora de risco relacionado ao câncer de bexiga superficial para a nossa população. Assim, esperamos que ela possa auxiliar urologistas a individualizar a decisão de condutas para cada paciente na prática clínica. 


\section{REFERÊNCIAS}

1. Torre LA, Bray F, Siegel RL, Ferlay J, Lortet-Tieulent J, Jemal A. Global cancer statistics, 2012. CA Cancer J Clin. 2015;65:87-108. doi: 10.3322/caac.21262.

2. Michaud DS, Clinton SK, Rimm EB, Willett WC, Giovannucci E. Risk of bladder cancer by geographic region in a U.S. cohort of male health professionals. Epidemiology. 2001;12:719-26.

3. Lynch CF, Cohen MB. Urinary system. Cancer. 1995;75(1 Suppl):316-29. doi: 10.1002/1097-0142(19950101) 75:1+<316::AID-CNCR2820751314>3.0.CO;2-T.

4. Scosyrev E, Noyes K, Feng C, Messing E. Sex and racial differences in bladder cancer presentation and mortality in the US. Cancer. 2009;115:68. doi: 10.1002/cncr.23986.

5. Parkin DM. The global burden of urinary bladder cancer. Scand J Urol Nephrol Suppl. 2008;218:12-20.

6. Lerner SP, Bajorin DF, Dinney CP, et al. Summary and Recommendations from the National Cancer Institute's Clinical Trials Planning Meeting on Novel Therapeutics for Non-Muscle Invasive Bladder Cancer. Bladder Cancer. 2016;2(2):165-202. doi:10.3233/BLC-160053.

7. Kluth LA, Black PC, Bochner BH, et al. Prognostic and prediction tools in bladder cancer: a comprehensive review of the literature. Eur Urol. 2015;68:238-53. doi: 10.1016/j. eururo.2015.01.032.

8. Chamie K, Saigal CS, Lai J, Hanley JM, Setodji CM, Konety BR, Litwin MS; Urologic Diseases in America Project. Compliance with guidelines for patients with bladder cancer: variation in the delivery of care. Cancer 2011;117(23):5392401. doi: 10.1002/cncr.26198.

9. Ross PL, Gerigk C, Gonen M, Yossepowitch O, Cagiannos I, Sogani PC, Scardino PT, Kattan MW. Comparisons of nomograms and urologists' predictions in prostate cancer. Semin Urol Oncol. 2002;20(2):82-8.

10. Specht MC, Kattan MW, Gonen M, Fey J, Van Zee KJ. Predicting nonsentinel node status after positive sentinel lymph biopsy for breast cancer: clinicians versus nomogram. Ann Surg Oncol. 2005;12:654-9. doi: 10.1245/ ASO.2005.06.037.

11. Edge SB, Compton CC. The American Joint Committee on Cancer: the 7th edition of the AJCC cancer staging manual and the future of TNM. Ann Surg Oncol 2010;17:1471-4. doi: 10.1245/s10434-010-0985-4.

12. Sylvester RJ, van der Meijden AP, Oosterlinck W, Witjes JA, Bouffioux C, Denis L, Newling DW, Kurth K. Predicting recurrence and progression in individual patients with stage Ta T1 bladder cancer using EORTC risk tables: a combined analysis of 2596 patients from seven EORTC trials. Eur Urol. 2006;49:466-75, discussion 475-7. doi: 10.1016/j. eururo.2005.12.031.

13. Hernandez V, De La Pena E, Martin MD, Blázquez C, Diaz FJ, Llorente C. External validation and applicability of the EORTC risk tables for non-muscle-invasive bladder cancer. World J Urol. 2011;29:409-14. doi: 10.1007/s00345-0100635-2.

Recebido em: 14.04.17

Aceito em: 17.04.17
14. Sakano S, Matsuyama H, Takai K, Yoshihiro S, Kamiryo Y, Shirataki S, Kaneda Y, Hashimoto O, Joko K, Suga A, Yamamoto M, Hayashida S, Baba Y, Aoki A; Yamaguchi Uro-Oncology Group. et al. Risk group stratification to predict recurrence after transurethral resection in Japanese patients with stage Ta and T1 bladder tumours: validation study on the European Association of Urology guidelines. BJU Int. 2011;107:1598-604. doi: 10.1111/j.1464-410X.2010.09850.x.

15. Seo KW, Kim BH, Park CH, Kim CI, Chang HS. The efficacy of the EORTC scoring system and risk tables for the prediction of recurrence and progression of non-muscleinvasive bladder cancer after intravesical bacillus calmetteguerin instillation. Korean J Urol. 2010;51:165-70. doi: 10.4111/kju.2010.51.3.165.

16. van Rhijn BWG, Zuiverloon TCM, Vis AN, Radvanyi F, van Leenders GJ, Ooms BC, Kirkels WJ, Lockwood GA, Boevé ER, Jöbsis AC, Zwarthoff EC, van der Kwast TH. Molecular grade (FGFR3/MIB-1) and EORTC risk scores are predictive in primary non-muscle-invasive bladder cancer. Eur Urol. 2010;58:433-41. doi: 10.1016/j.eururo.2010.05.043.

17. Pillai R, Wang D, Mayer EK, Abel P. Do standardised prognostic algorithms reflect local practice? Application of EORTC risk tables for non-muscle invasive ( $\mathrm{pTa} / \mathrm{pT} 1)$ bladder cancer recurrence and progression in a local cohort. Scientific World J. 2011;11:751-9. doi: 10.1100/tsw.2011.77.

18. Fernandez-Gomez J, Madero R, Solsona E, Unda M, Martinez-Piñeiro L, Ojea A, Portillo J, Montesinos M, Gonzalez M, Pertusa C, Rodriguez-Molina J, Camacho JE, Rabadan M, Astobieta A, Isorna S, Muntañola P, Gimeno A, Blas M, Martinez-Piñeiro JA; Club Urológico Español de Tratamiento Oncológico. The EORTC tables overestimate the risk of recurrence and progression in patients with non-muscle-invasive bladder cancer treated with bacillus Calmette-Guerin: external validation of the EORTC risk tables. Eur Urol 2011;60:423-30. doi: 10.1016/j. eururo.2011.05.033.

19. Fernandez-Gomez J, Madero R, Solsona E, Unda M, Martinez-Piñeiro L, Gonzalez M, Portillo J, Ojea A, Pertusa C, Rodriguez-Molina J, Camacho JE, Rabadan M, Astobieta A, Montesinos M, Isorna S, Muntañola P, Gimeno A, Blas M, Martinez-Piñeiro JA. Predicting nonmuscle invasive bladder cancer recurrence and progression in patients treated with bacillus Calmette-Guerin: the CUETO scoring model. J Urol. 2009;182:2195-203. doi: 10.1016/j.juro.2009.07.016.

20. Rosevear HM, Lightfoot AJ, Nepple KG, O’Donnell MA. Usefulness of the Spanish Urological Club for Oncological Treatment scoring model to predict nonmuscle invasive bladder cancer recurrence in patients treated with intravesical bacillus Calmette-Guerin plus interferon-alpha. J Urol. 2011;185:67-71. doi: 10.1016/j.juro.2010.08.083.

21. Xylinas E, Kent M, Kluth L, Pycha A, Comploj E, Svatek RS, Lotan Y, Trinh QD, Karakiewicz PI, Holmang S, Scherr DS, Zerbib M, Vickers AJ, Shariat SF. Accuracy of the EORTC risk tables and of the CUETO scoring model to predict outcomes in nonmuscle-invasive urothelial carcinoma of the bladder. Br J Cancer. 2013;109:1460-6. doi: 10.1038/ bjc.2013.372. 